\section{Lesões intra-epiteliais cervicais em adolescentes: estudo dos achados citológicos entre 1999 e 2005, no Município do Rio de Janeiro, Brasil}

\author{
Cervical intraepithelial lesions in adolescents: \\ cytological findings from 1999 to 2005 in \\ Rio de Janeiro, Brazil
}

\author{
${ }^{1}$ Escola Nacional de Saúde \\ Pública Sergio Arouca, \\ Fundação Oswaldo Cruz, \\ Rio de Janeiro, Brasil. \\ 2 Universidade Federal do \\ Rio de Janeiro, Rio de Janeiro, \\ Brasil. \\ Correspondência \\ M. L. Pedrosa \\ Escola Nacional de Saúde \\ Pública Sergio Arouca, \\ Fundação Oswaldo Cruz. \\ Rua Tonelero 30, apto. 203 , \\ Rio de Janeiro, $R J$ \\ 22030-000, Brasil. \\ michelelpedrosa@gmail.com
}

\begin{abstract}
The prevalence of cervical intraepithelial neoplasias has increased in adolescents, probably due to the decrease in age at first intercourse and increase in the number of sexual partners. Our objective was to analyze the trends in cervical intraepithelial lesions in adolescents in the city of Rio de Janeiro, Brazil. We thus conducted a retrospective analysis of 1,516,407 Pap smears performed at public health units from 1999 to 2005. During the study period, the distribution and temporal trend in cytological abnormalities was compared between adolescent and adult women. There were more atypical smears in adolescents, mainly low-grade lesions. In adolescents, cervical abnormalities doubled during this period, from $6.4 \%$ to $12.4 \%$, while in adult women it increased from $4.5 \%$ to $6.7 \%$. Statistical analysis showed a tendency towards a linear increase in frequency of abnormal Pap smears in both adolescents and adults $(0.008 \%$ and $0.003 \%$, respectively). Based on this and other studies, we recommend including adolescents in the target population of the Brazilian cervical cancer screening program ("Programa Viva Mulher").
\end{abstract}

Uterine Cervical Neoplasms; Adolescent; Women's Health
Michele Lopes Pedrosa 1,2 Inês Echenique Mattos 1 Rosalina Jorge Koifman 1

\section{Introdução}

O câncer do colo do útero corresponde à segunda causa de óbito por neoplasia em mulheres no mundo, ocorrendo mais de 250 mil óbitos anualmente 1 . No Brasil, há elevada freqüência dessa neoplasia, tendo sido descritas, em 2002, taxas padronizadas de incidência para as cidades de Campinas (São Paulo) e Goiânia (Goiás), respectivamente de 14,1 e 38,2 por $100 \mathrm{mil} \mathrm{mu-}$ lheres 2 .

A infecção por subtipos oncogênicos do papilomavírus humano (HPV) constitui o fator causal primário para o desenvolvimento da neoplasia invasora do colo uterino 3,4 , tendo sido identificada em quase a totalidade dos carcinomas cervicais 5 . Esse vírus é adquirido através de transmissão sexual, freqüentemente em faixas etárias jovens, constituindo uma das doenças sexualmente transmissíveis (DSTs) mais comuns entre adolescentes, com prevalência de $30 \%$ a $50 \%$ nessa faixa etária 6 .

O desenvolvimento normalmente lento do câncer de colo uterino permite a identificação, por meio da citologia corada pelo método de $\mathrm{Pa}$ panicolaou, de lesões precursoras, denominadas lesões intra-epiteliais ou neoplasias intra-epiteliais cervicais (NIC) 1. Essas lesões são classificadas de acordo com a maior ou menor probabilidade de evolução para câncer em, respectivamente, lesões de baixo grau (NIC I) e lesões de alto grau (NIC II/NIC III) 7 . 
Vários estudos sugeriram que a freqüência das alterações citológicas cervicais vem crescendo entre a população de adolescentes 8,9,10,11,12. A mudança de padrões de comportamento nesse grupo, com diminuição da idade de início da vida sexual e aumento no número de parceiros sexuais, associa-se a maior probabilidade de infecção pelo HPV 13,14, contribuindo para aumentar o risco de desenvolvimento de lesões pré-neoplásicas e invasivas da cérvice uterina. Há ainda evidências que as adolescentes e as mulheres jovens são mais vulneráveis à infecção pelo HPV por razões biológicas 15. Embora predominem as lesões de baixo grau entre as adolescentes, há risco de desenvolvimento de alterações com maior grau de displasia naquelas portadoras de subtipos oncogênicos 16 .

O aumento da freqüência de lesões pré-neoplásicas cervicais em adolescentes aponta para a necessidade de investigação do comportamento dessas alterações nessa faixa etária, cuja compreensão poderá auxiliar o desenvolvimento de mecanismos de intervenção que reduzam as taxas de morbi-mortalidade por essa neoplasia. $\mathrm{O}$ objetivo deste estudo foi analisar a distribuição e a tendência temporal das alterações citológicas cervicais em adolescentes do Município do Rio de Janeiro, Brasil, nos anos de 1999 a 2005, comparando-se os dados deste grupo com os achados em mulheres adultas.

\section{Metodologia}

Realizou-se estudo exploratório retrospectivo de exames colpocitológicos registrados no banco de dados do Serviço Integrado Tecnológico em Citopatologia (SITEC), pertencente ao Instituto Nacional de Câncer (INCA). Identificaram-se 1.516.407 citologias coletadas, no período de 1o de janeiro de 1998 a 31 de dezembro de 2005, em munícipes da cidade do Rio de Janeiro, que constituíram a população de estudo deste trabalho. Para tanto, utilizou-se o programa de tabulação de dados desenvolvido pelo INCA para a produção de informações a partir do referido banco, sendo selecionados os filtros "idade" (10 a 19 anos e 20 ou mais anos), "município de residência" (Rio de Janeiro) e "ano da coleta” (1999 a 2005).

As citologias analisadas pelo SITEC foram provenientes de unidades do Sistema Único de Saúde (SUS) e coradas pela técnica de Papanicolaou. O diagnóstico seguiu o sistema de classificação citológica de Richart 17: normal; atipias escamosas de significado indeterminado (ASCUS); atipias glandulares de significado indeterminado (AGUS); infecção pelo HPV; neoplasia intra-epitelial cervical grau 1 (NIC I), neoplasia intra-epitelial cervical grau 2 (NIC II), neoplasia intra-epitelial cervical grau 3 (NIC III); carcinoma escamoso invasor. Consideraram-se adolescentes as mulheres entre 10 e 19 anos, seguindo a classificação da Organização Mundial da Saúde (OMS) 18, e mulheres adultas aquelas com idade igual ou superior a 20 anos. As citologias foram classificadas segundo essas faixas etárias. Para a análise de tendência temporal, optou-se por agrupar os diagnósticos, de acordo com a probabilidade de evolução para doença invasora, em lesões de baixo grau (LSIL), correspondendo à infecção pelo HPV e NIC I, e lesões de alto grau (HSIL), relacionando-se com diagnóstico de NIC II ou NIC III 19.

A análise estatística foi realizada com auxílio do SPSS para Windows versão 14 (SPSS Inc., Chicago, Estados Unidos) e do Excel 2002 (Microsoft Corp., Estados Unidos). Foram calculadas as medidas de tendência central e de dispersão da variável idade no grupo de adolescentes. Através da construção de tabelas de freqüências, comparou-se, ao longo do período, a distribuição das alterações citológicas entre adolescentes e mulheres adultas. Para avaliar a presença de homogeneidade na distribuição, foram aplicados testes com uso da distribuição qui-quadrado, bicaudal, considerando nível de 95\% de significância.

Explorou-se a relação entre a freqüência de alterações citológicas segundo a idade em adolescentes entre 15 e 19 anos, considerando, como variáveis dependentes $(\mathrm{Y})$, a prevalência de lesões em cada categoria do diagnóstico citológico de Richart e, como variável independente $(\mathrm{X})$, a idade da adolescente no momento da coleta do material cervical para exame.

Analisou-se a tendência temporal de distribuição das alterações citológicas cervicais em adolescentes e mulheres adultas. Foram estimados três modelos considerando-se como variáveis dependentes $(\mathrm{Y})$ : a prevalência de alterações citológicas, a prevalência de lesão de baixo grau e a prevalência de lesão de alto grau. A variável independente $(\mathrm{X})$, nos três modelos, foi o ano da coleta da citologia.

Para cada modelo, foram construídos, inicialmente, diagramas de dispersão entre as variáveis independentes e as variáveis dependentes para visualizar a função que melhor poderia expressar a relação entre elas. A presença e o grau de associação entre as variáveis dependentes e independentes foram verificados através do cálculo do coeficiente de correlação de Pearson. O valor de $\mathrm{p}<0,05$ foi considerado estatisticamente significativo.

Para análise de tendência, optou-se pela utilização da técnica estatística de regressão linear simples, devido à facilidade de elaboração e 
interpretação e ao poder estatístico dessa modelagem. Para evitar a autocorrelação entre os termos da equação de regressão, foi utilizado o artifício de centralizar a variável ano, transformando-a em ano calendário menos 2002 (ano -2002), pois este era o ponto médio da série histórica. O modelo linear foi definido como $\mathrm{Y}=\beta_{0}$ $+\beta_{1} \mathrm{x}$, sendo $\beta_{0}$ a prevalência média do período e $\beta_{1}$ o incremento (acréscimo ou decréscimo) médio do período. Admitiu-se tendência linear estatisticamente significativa somente quando a sua probabilidade de ter ocorrido foi igual ou menor do que 0,05 .

O projeto de pesquisa Controle do Câncer de Colo de Útero foi aprovado pelo Comitê de Ética em Pesquisa da Escola Nacional de Saúde Pública Sergio Arouca, Fundação Oswaldo Cruz.

\section{Resultados}

No período de 1999 a 2005, foram registradas, no banco de dados do SITEC, 1.516.407 citologias, $10 \%$ em mulheres na faixa etária entre 10 e 19 anos. Identificaram-se 89.665 exames alterados (5,9\% do total de citologias), $15 \%$ dos quais em adolescentes.

No grupo de adolescentes, a média de idade foi de 17,2 anos (desvio-padrão = 1,6), e a mediana, de 17,0 anos. Identificaram-se 15.878 adolescentes entre 10 e 14 anos, correspondendo a $10,4 \%$ do total de adolescentes do banco.

Considerando-se o conjunto das alterações citológicas, observou-se maior prevalência de anormalidades citológicas no grupo de adolescentes $(8,9 \%)$ do que no grupo de mulheres adultas (5,6\%). Na faixa etária de 10 a 14 anos, foi encontrado 5,5\% de exames anormais, inferior ao percentual relativo a mulheres entre 15 e 19 anos $(9,3 \%)$ (Tabela 1$)$.

A análise da distribuição das alterações citológicas em adolescentes e adultas no período de estudo demonstrou proporções distintas em todas as categorias diagnósticas ( $\mathrm{p}<0,01)$. As lesões de baixo grau corresponderam a mais da metade das alterações cervicais no grupo de adolescentes, sendo 2,9 vezes mais freqüente nesse grupo do que entre as mulheres adultas. Observou-se prevalência 1,2 vez superior de alterações de alto grau entre as adultas do que entre as adolescentes, respectivamente $0,99 \% \mathrm{e}$ $0,82 \%$. No grupo de adolescentes, mais de $90 \%$ das alterações de alto grau corresponderam a NIC II, e o percentual de NIC III foi seis vezes menor que o calculado para as mulheres adultas. No subgrupo entre 10 e 14 anos, a maior parte das citologias alteradas corresponderam a alterações de baixo grau, sendo de $0,02 \%$ a freqüência de alterações de alto grau, inferior às demais faixas etárias avaliadas (Tabela 1).

Entre 1999 e 2005, a prevalência de alterações cervicais em adolescentes duplicou, passando de $6,4 \%$ para $12,4 \%$, enquanto, nas mulheres adultas, aumentou de $4 \%$ para $6,1 \%$. Ao se analisarem as lesões de baixo grau, observou-se elevação de $3,5 \%$ para $6,5 \%$ nas adolescentes e de $1,3 \%$ para $2,4 \%$ nas mulheres adultas. Demonstrou-se, no período estudado, tendência

Distribuição dos diagnósticos citológicos entre adolescentes (10 a 14 anos e 15 a 19 anos) e mulheres adultas identificadas no banco de dados do Serviço Integrado Tecnológico em Citopatologia (SITEC) no período de 1999 a 2005.

\begin{tabular}{|c|c|c|c|c|c|c|c|c|c|}
\hline \multirow[t]{3}{*}{ Mulheres } & \multirow{3}{*}{$\begin{array}{c}\text { Citologias } \\
\text { normais }\end{array}$} & \multicolumn{7}{|c|}{ Citologias alteradas } & \multirow{3}{*}{$\begin{array}{c}\text { Total de } \\
\text { citologias }\end{array}$} \\
\hline & & \multirow[t]{2}{*}{ ASCUS } & \multirow[t]{2}{*}{ AGUS } & \multirow{2}{*}{$\begin{array}{c}\text { Baixo grau } \\
\text { NIC I }\end{array}$} & \multicolumn{2}{|c|}{ Alto grau } & \multirow[t]{2}{*}{ Câncer } & \multirow[t]{2}{*}{ Total } & \\
\hline & & & & & NIC II & NIC III & & & \\
\hline \multirow[t]{2}{*}{ Adolescentes } & 138.805 & 5.031 & 97 & 7.092 & 1.178 & 74 & 0 & 13.472 & 152.277 \\
\hline & $(91,15 \%)$ & $(3,30 \%)$ & $(0,06 \%)$ & $(4,66 \%)$ & $(0,77 \%)$ & $(0,05 \%)$ & $(0,0 \%)$ & $(8,85 \%)$ & $(100,00 \%)$ \\
\hline \multirow[t]{2}{*}{ 10-14 anos } & 15.013 & 306 & 4 & 494 & 58 & 3 & 0 & 865 & 15.878 \\
\hline & $(94,55 \%)$ & $(1,93 \%)$ & $(0,03 \%)$ & $(3,11 \%)$ & $(0,37 \%)$ & $(0,02 \%)$ & $(0,0 \%)$ & $(5,45 \%)$ & $(100,00 \%)$ \\
\hline \multirow[t]{2}{*}{ 15-19 anos } & 123.792 & 4.725 & 93 & 6.598 & 1.120 & 71 & 0 & 12.607 & 136.399 \\
\hline & $(90,76 \%)$ & $(3,46 \%)$ & $(0,07 \%)$ & $(4,84 \%)$ & $(0,82 \%)$ & $(0,05 \%)$ & $(0,0 \%)$ & $(9,25 \%)$ & $(100,00 \%)$ \\
\hline \multirow[t]{2}{*}{ Adultas } & 1.287 .937 & 36.510 & 4.447 & 21.514 & 8.349 & 4.556 & 817 & 76.193 & 1.364 .130 \\
\hline & $(94,41 \%)$ & $(2,68 \%)$ & $(0,33 \%)$ & $(1,58 \%)$ & $(0,61 \%)$ & $(0,33 \%)$ & $(0,06 \%)$ & $(5,59 \%)$ & $(100,00 \%)$ \\
\hline \multirow[t]{2}{*}{ Total } & 1.426 .742 & 41.541 & 4.544 & 28.606 & 9.527 & 4.630 & 817 & 89.665 & 1.516 .407 \\
\hline & $(94,09 \%)$ & $(2,74 \%)$ & $(0,30 \%)$ & $(1,89 \%)$ & $(0,63 \%)$ & $(0,31 \%)$ & $(0,05 \%)$ & $(5,91 \%)$ & $(100,00 \%)$ \\
\hline
\end{tabular}

AGUS: atipias glandulares de significado indeterminado; ASCUS: atipias escamosas de significado indeterminado; NIC: neoplasias intra-epiteliais cervicais. 
de crescimento linear anual na freqüência de alterações citológicas cervicais em ambos os grupos. Entre as adolescentes, estimou-se aumento anual médio de $0,008 \%$ na proporção de alterações citológicas cervicais, mais acentuado do que nas mulheres adultas para as quais foi calculado incremento anual de 0,003\%. Da mesma forma, foi identificada tendência de crescimento linear das alterações de baixo grau, mais pronunciada no grupo de adolescentes $\left(\beta_{1}=0,004\right)$ do que no grupo de adultas $\beta_{1}=$ 0,002). Observou-se estabilidade na prevalência das lesões de alto grau no período estudado em ambos os grupos (Figura 1; Tabela 2).

Figura 1

Curvas de tendência linear da prevalência de alterações citológicas em adolescentes e mulheres adultas identificadas no banco de dados do Serviço Integrado Tecnológico em Citopatologia (SITEC), no período de 1999 a 2005.

1a) Mulheres adolescentes

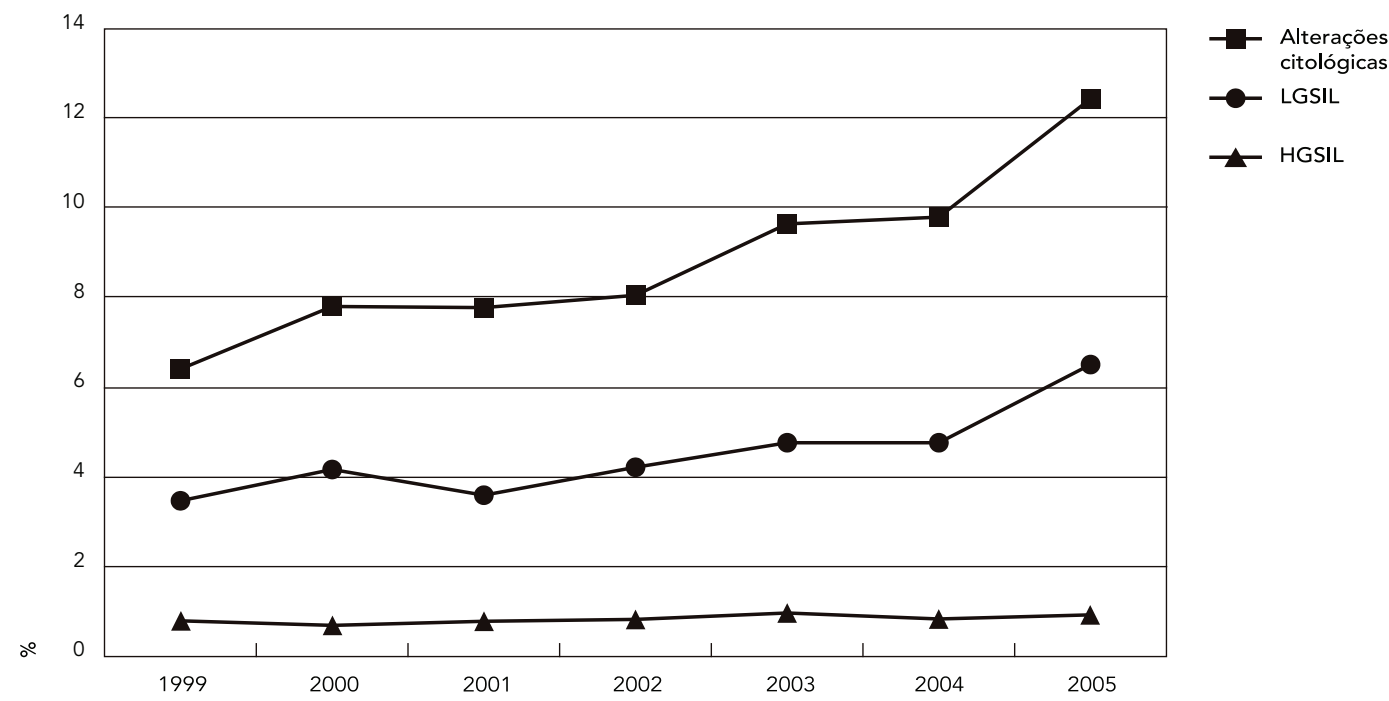

1b) Mulheres adultas

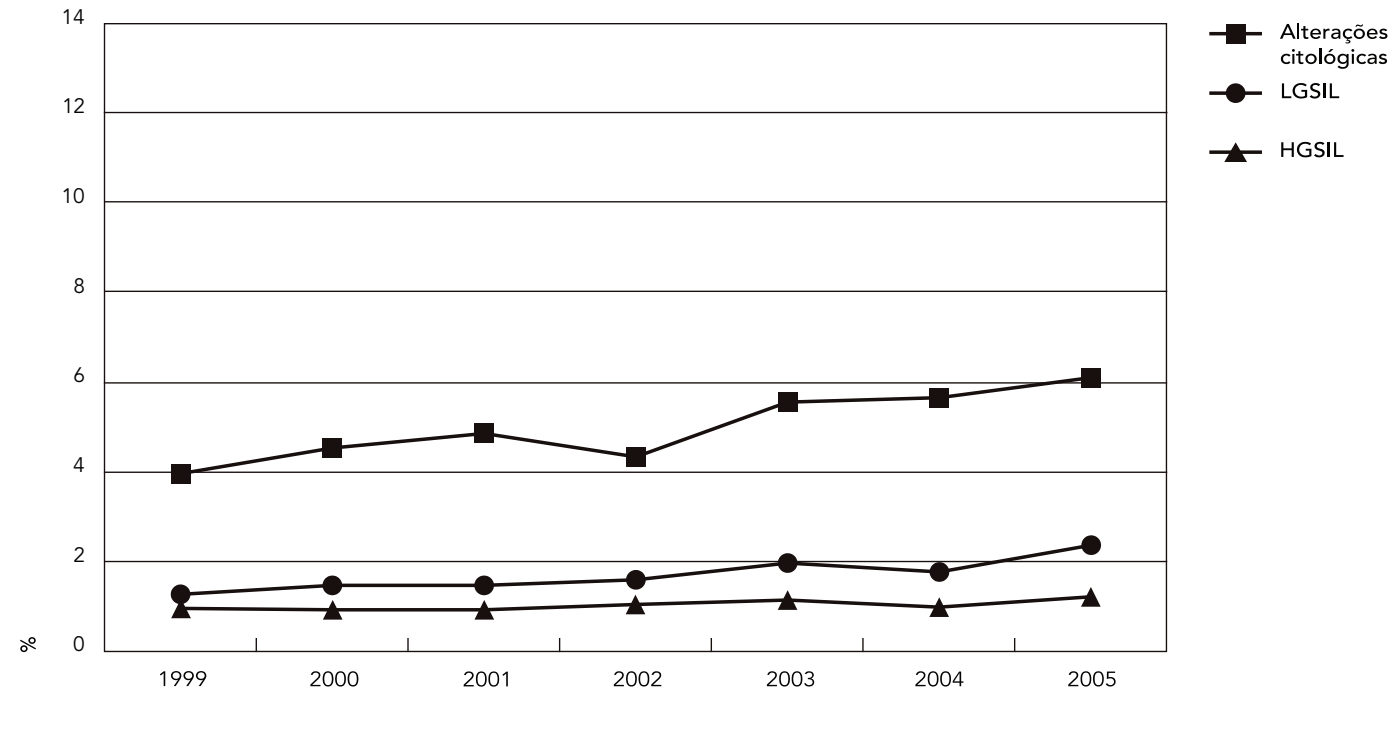

LGSIL: lesões intra-epiteliais de baixo grau (NIC I); HGSIL: lesões intra-epiteliais de alto grau (NIC II/NIC III). 
Modelos de regressão linear: prevalências do conjunto das alterações citológicas cervicais e prevalências de alterações de baixo e alto grau em adolescentes e mulheres adultas identificadas no banco de dados do Serviço Integrado Tecnológico em Citopatologia (SITEC), no período de 1999 a 2005.

\begin{tabular}{lcccc}
\hline Mulheres & Prevalência & $\mathbf{R}^{2}$ & Modelo & $\mathbf{p}$ * \\
\hline \multirow{2}{*}{ Adolescentes } & Alterações citológicas & 0,88 & $Y=0,0887+0,0085(x-2002)$ & 0,002 \\
& Alterações de baixo grau & 0,76 & $Y=0,0452+0,0041(x-2002)$ & 0,011 \\
Adultas & Alterações de alto grau & 0,53 & $Y=0,0071+0,0003(x-2002)$ & 0,064 \\
& Alterações citológicas & 0,84 & $Y=0,0501+0,0033(x-2002)$ & 0,004 \\
& Alterações de baixo grau & 0,83 & $Y=0,0171+0,0016(x-2002)$ & 0,004 \\
& Alterações de alto grau & 0,58 & $Y=0,0103+0,0004(x-2002)$ & 0,051 \\
\hline
\end{tabular}

R2: coeficiente de determinação.

* Nível descritivo do coeficiente $\beta_{1}$.

A análise da distribuição das alterações citológicas pela idade no momento da coleta, no grupo entre 15 a 19 anos, demonstrou um predomínio de lesões de baixo grau em todas as idades, variando entre $49,4 \%$ e $54,7 \%$. Observou-se associação linear positiva entre a idade no momento da coleta e a prevalência de lesões de alto grau (coeficiente de correlação: 0,99; $p=0,001$ ) e associação linear negativa entre a idade no momento da coleta e a prevalência de lesões de baixo grau (coeficiente de correlação: $-0,88 ; \mathrm{p}=0,05$ ). A presença de atipias indeterminadas não se correlacionou com a idade no momento da coleta (Tabela 3).

\section{Discussão}

No presente estudo, a distribuição das alterações citológicas cervicais pelo total de exames identificou, nas adolescentes, $4,7 \%$ de alterações de baixo grau e $0,8 \%$ de alterações de alto grau. Apesar de os percentuais de alterações intra-epiteliais entre adolescentes diferirem na literatura, relatando-se prevalência entre $1 \%$ e $14 \%$ para baixo grau e entre $0,3 \%$ e 1,3\% para alto grau, há concordância quanto à elevada freqüência de anormalidades citológicas cervicais nessa faixa etária, com predomínio de alterações de baixo grau 9,10,20,21,22,23. Essa variação possivelmente é conseqüência das características da população avaliada nos estudos, da diversidade das técnicas de coleta utilizadas e, sobretudo, da diferença nos critérios diagnósticos entre laboratórios.

Nenhum caso de câncer cervical foi identificado em nosso trabalho, o que era esperado, visto ser a malignidade cervical rara na adolescência. O Surveillance, Epidemiology, and End Results 24
(National Cancer Institute Estados Unidos) estimou, para o período de 1975 a 2000, incidência acumulada de câncer de colo uterino de 0,4 por 100 mil em mulheres norte-americanas entre 10 e 19 anos.

No presente estudo, observou-se tendência de crescimento linear das alterações cervicais, no período entre 1999 e 2005, em ambos os grupos. Nas adolescentes esse acréscimo foi, em média, 2,6 vezes maior nas adolescentes do que nas adultas. O incremento anual médio do diagnóstico de lesões de baixo grau em adolescentes no período estudado foi 2,5 vezes superior ao incremento anual médio nas mulheres adultas.

A comparação dos achados de dois grandes estudos realizados nos Estados Unidos, com intervalo de quase duas décadas, avaliando adolescentes com características demográficas e socioeconômicas semelhantes, demonstra crescimento na detecção de alterações citológicas cervicais. Sadeghi et al. 25 analisaram 194.069 citologias registradas, no ano de 1981, no banco de dados do Serviço de Rastreamento de Câncer, relativas a mulheres entre 15 e 19 anos. Foram identificadas $1,8 \%$ de alterações epiteliais, sendo $1,5 \%$ de baixo grau e 0,3\% de alto grau. Em 1999, Mount \& Papillo 8, revisando 10.296 exames citológicos cervicais de mulheres entre 10 e 19 anos de idade coletados durante o período de um ano, encontraram $3,2 \%$ de lesões intra-epiteliais cervicais, $2,5 \%$ correspondendo a alterações de baixo grau e $0,7 \%$ a alterações de alto grau. Observou-se incremento de 1,8 vez na prevalência global de lesões cervicais, sendo que as alterações de alto grau se elevaram em mais de duas vezes.

Aumento crescente da prevalência de lesões cervicais em adolescentes nas últimas décadas, em freqüência superior àquela encontrada nas 
Distribuição das alterações citológicas pela idade no momento da coleta do material cervical, entre adolescentes de 15 a 19 anos identificadas no banco de dados do Serviço Integrado Tecnológico em Citopatologia (SITEC), no período de 1999 a 2005.

\begin{tabular}{|c|c|c|c|c|c|c|c|}
\hline \multicolumn{3}{|c|}{ Diagnóstico citológico } & \multicolumn{3}{|l|}{ Idade em anos } & \multicolumn{2}{|c|}{ Correlação linear } \\
\hline & 15 & 16 & 17 & 18 & 19 & $\begin{array}{l}\text { Coeficiente de } \\
\text { correlação }\end{array}$ & $\begin{array}{l}\text { Valor } \\
\text { de } p\end{array}$ \\
\hline AGUS & $8(0,6 \%)$ & $9(0,4 \%)$ & $21(0,8 \%)$ & $24(0,8 \%)$ & $31(0,9 \%)$ & 0,79 & 0,11 \\
\hline ASCUS & $509(38,4 \%)$ & 767 (36,7\%) & $1.008(36,5 \%)$ & $1.140(37,2 \%)$ & $1.301(38,7 \%)$ & 0,17 & 0,78 \\
\hline Baixo grau & $716(54,0 \%)$ & $1.143(54,7 \%)$ & $1.489(54,0 \%)$ & $1.587(51,8 \%)$ & $1.663(49,4 \%)$ & $-0,88$ & 0,05 \\
\hline Alto grau & 92 (6,9\%) & 173 (8,3\%) & 242 (8,8\%) & $313(10,2 \%)$ & $371(11,0 \%)$ & 0,97 & 0,001 \\
\hline Total & $1.325(100,0 \%)$ & $2.092(100,0 \%)$ & $2.760(100,0 \%)$ & $3.064(100,0 \%)$ & $3.366(100,0 \%)$ & - & - \\
\hline
\end{tabular}

AGUS: atipias glandulares de significado indeterminado; ASCUS: atipias escamosas de significado indeterminado.

mulheres adultas, foi descrito em outros estudos realizados no Brasil. Utagawa et al. 26, ao estudarem citologias cervicais avaliadas pelo Instituto Adolfo Lutz, em São Paulo, entre 1987 e 1995, encontraram tendência de aumento linear na freqüência de anormalidades cervicais, maior no grupo de adolescentes $\left(\beta_{1}=0,12 \pm 0,06\right)$ do que entre mulheres adultas $\left(\beta_{1}=0,08 \pm 0,02\right)$. Longatto Filho et al. 11, complementando o estudo anterior com a inclusão das citologias avaliadas no Instituto Adolfo Lutz entre 1996 e 2001, demonstraram tendência a aumento linear significativo nesse período em ambos os grupos (adolescentes: $\beta_{1}=0,28 \pm 0,07$; adultas: $\beta_{1}=0,15 \pm 0,04$ ). Também Silva et al. 10, analisando os achados de citologias cervicais coletadas em mulheres de baixa renda residentes no Município de Uberaba e regiões adjacentes entre os períodos 1987-1991 e 1996-1999, observaram: aumento na freqüência de NIC I entre adolescentes (de $0,8 \%$ para $2,2 \%$ ) e mulheres adultas (de $0,7 \%$ para $1,2 \%$ ) e elevação do percentual de NIC II entre adolescentes (de $0,2 \%$ para $0,5 \%$ ).

$\mathrm{O}$ incremento observado no diagnóstico de lesões pré-neoplásicas cervicais em adolescentes explica-se, em grande parte, pela elevada prevalência da infecção pelo HPV nessa faixa etária 16. O aumento da infecção por esse vírus, assim como a presença de outras DSTs, relaciona-se, possivelmente, à mudança no comportamento sexual desse grupo, em especial ao aumento no número de parceiros sexuais e à precocidade da primeira relação sexual 27,28. O desenvolvimento de lesões intra-epiteliais cervicais parece ser diretamente proporcional ao número de parceiros referido pelas adolescentes 29 , possivelmente devido ao aumento da probabilidade de infecção pelo HPV conseqüente à maior exposição sexual 12,14. Kahn et al. 30 demonstraram que cada ano a menos entre a menarca e o primeiro coito associava-se a $12 \%$ de aumento na probabilidade de infecção pelo HPV, e que a redução da idade no primeiro coito em um ano relacionava-se a $20 \%$ de acréscimo no risco de infecção por esse vírus.

Parece existir, ainda, aumento de suscetibilidade da cérvice uterina das adolescentes à infecção pelo HPV relacionado à presença de maior percentual de células metaplásicas no colo uterino destas do que na cérvice das mulheres adultas. As células metaplásicas possuem elevada taxa de mitose, sendo capazes de dar suporte à replicação desse vírus 31. A persistência da infecção por subtipos oncogênicos do HPV é capaz de interferir no controle da replicação celular, acarretando, lentamente, modificações intra-epiteliais 32 que podem originar alterações pré-neoplásicas e invasoras 3,33 .

Outros mecanismos, que possivelmente podem contribuir para elevar a probabilidade de aquisição de DSTs, incluem maior suscetibilidade ao trauma durante a relação sexual e o desenvolvimento incompleto das respostas imunes locais. A produção diminuída de muco cervical, barreira protetora contra diversos agentes infecciosos, ocasionada pela ausência de progesterona cíclica resultante de ciclos anovulatórios no início da puberdade, também pode estar envolvida 30.

Provavelmente, a associação positiva observada entre presença de lesões de alto grau e a idade cronológica se relacione ao período de tempo decorrido desde a infecção inicial, propiciando o desenvolvimento de displasias mais acentuadas. Em nosso estudo, a idade no momento da coleta do material citológico mostrou forte correlação positiva com o diagnóstico de lesão de alto grau. Esse achado é corroborado pelo estudo de Wright et al. ${ }^{34}$, no qual, identificou-se o dobro de lesões 
de alto grau em adolescentes com 18 anos de idade quando comparadas àquelas com 15 anos de idade. Da mesma forma, a análise dos resultados histopatológicos de biópsias obtidas do colo uterino de adolescentes atendidas em um hospital público do Rio de Janeiro 35 demonstrou ser a probabilidade de diagnosticar alterações de alto grau entre mulheres com idade maior ou igual a 17 anos 5,3 vezes maior (IC95\%: 1,8-15,6) do que nas de idade inferior.

A maior parte das lesões pré-neoplásicas identificadas em adolescentes correspondem a lesões de baixo grau, representando as alterações citológicas conferidas pela presença do HPV no interior da célula cervical 7 e que apresentam elevado percentual de regressão espontânea, estimada em $91 \%$ por Moscicki et al. 36 em três anos de seguimento. Entretanto, em um percentual significativo dessa população, foram observadas lesões de alto grau, indicando provável infecção por subtipos oncogênicos do HPV, o que confere risco aumentado de desenvolvimento de neoplasia invasora cervical, visto que a infecção persistente por esses subtipos é fator necessário para gênese dessa doença 37 .

No Brasil, em 1996, o Ministério da Saúde implantou o Programa Nacional de Controle do Câncer do Colo do Útero e Mama ("Programa Viva Mulher") 38 com objetivo de reduzir as taxas de morbi-mortalidade por essas neoplasias. As estratégias de prevenção e controle do câncer de colo uterino baseiam-se no rastreamento das lesões precursoras por meio do exame citopatológico, tendo como grupo prioritário mulheres entre 25 e 49 anos.

A justificativa para a não inclusão de mulheres mais jovens na população alvo é a menor eficiência dessas ações nessa faixa etária, devido ao predomínio de lesões de baixo grau com elevada taxa de regressão. Logo, a possível identificação de lesões de pouca importância clínica e a resolução espontânea poderiam ocasionar custos adicionais desnecessários 39,40.

A recomendação da International Agency for Research on Cancer ${ }^{41}$, pertencente à OMS, é que cada país defina a idade de início do rastreamento de acordo com a prevalência da infecção pelo HPV, carcinoma invasor e lesões precursoras por faixa etária. Entre as adolescentes, embora o percentual de neoplasia invasora seja desprezível, observa-se elevado percentual delesões intra-epiteliais cervicais, com considerável parcela de lesões de alto grau. Adicionalmente, é possível que as adolescentes apresentem um tempo de latência entre a infecção pelo HPV e o desenvolvimento de lesões cervicais mais cur- to do que o usualmente descrito para mulheres adultas 42,43 . Tendo em vista esses argumentos, a American Academy of Pediatrics recomenda oferta de exame ginecológico, incluindo rastreamento do câncer cervical, a todas as adolescentes que se tornam sexualmente ativas ${ }^{44}$.

A população avaliada em nosso estudo representa uma importante amostra da população usuária do sistema público de saúde na cidade do Rio de Janeiro, visto ser o laboratório do SITEC responsável pela análise de aproximadamente $90 \%$ dos exames citológicos cervicais realizados nessa rede (Departamento de Informática do SUS. http://datasus.gov.br/cgi/sia/ pamap.htm, acessado em 26/Mai/2007). Para o Município do Rio de Janeiro, estima-se que cerca de $50 \%$ da população seja usuária do SUS 45 , constituída, em sua maior parte, por população de baixa renda. Assim, os resultados desse trabalho devem aproximar-se aos valores reais dessa classe populacional, apresentando-se como uma referência para o planejamento, abordagem e seguimento das atividades de rastreio do câncer cervical em nosso meio. Não se pode, entretanto, generalizar os achados a todas as adolescentes residentes no município, visto que o padrão sócio-econômico influencia fatores como práticas sexuais, função imune e acesso a cuidados de saúde.

É importante ressaltar, ainda, que as citologias avaliadas em nosso estudo foram obtidas de mulheres que procuraram atendimento em unidades de saúde. Sintomas relacionados a doenças sexualmente transmissíveis representam uma das principais causas, relacionada à saúde reprodutiva, de utilização do sistema de saúde por adolescentes 46,47 . Assim, é possível que essas adolescentes constituam um subgrupo com maior probabilidade de ser portador de DSTs, incluindo o HPV.

Baseado neste e em outros estudos, sugerimos a implementação de estratégias de prevenção e controle voltadas para adolescentes que iniciaram vida sexual, incluindo o rastreio citológico periódico. A educação sexual pode ser um importante caminho para prevenir a infecção pelo HPV e o desenvolvimento de lesões precursoras do câncer de colo uterino. Informar e conscientizar os adolescentes sobre o HPV e os riscos associados, assim como sobre as formas de prevenção, possivelmente contribuirá para reduzir a contaminação por esse vírus e aumentar o diagnóstico das lesões intra-epiteliais existentes, possibilitando seu controle e influenciando na diminuição da incidência do câncer de colo uterino em longo prazo. 


\section{Resumo}

A freqüência das lesões precursoras do câncer cervical vem crescendo entre a população de adolescentes, possivelmente, em conseqüência da diminuição da idade de início da vida sexual e do aumento no número de parceiros sexuais. Com o objetivo de analisar o comportamento das lesões precursoras do câncer cérvico-uterino entre adolescentes do Município do Rio de Janeiro, Brasil, estudou-se, de forma retrospectiva, 1.516.407 exames citológicos provenientes de unidades do Sistema Único de Saúde entre 1999 e 2005. Foram comparadas, ao longo do período, a distribuição das alterações citológicas e a tendência temporal dessa distribuição entre adolescentes e mulheres adultas. No período, observou-se maior freqüência de anormalidades citológicas no grupo de adolescentes, predominando as lesões de baixo grau. A prevalência de alterações cervicais em adolescentes duplicou, passando de $6,4 \%$ para $12,4 \%$, enquanto, nas mulheres adultas, aumentou de $4 \%$ para $6,1 \%$, sendo estimado incremento anual médio entre as adolescentes e mulheres adultas de, respectivamente $0,008 \%$ e $0,003 \%$. Com base neste estudo e na literatura consultada, sugerimos a inclusão das adolescentes no grupo prioritário para rastreio citológico periódico do "Programa Viva Mulher".

Neoplasias do Colo do Útero; Adolescente; Saúde da Mulher

\section{Referências}

1. International Agency for Research on Cancer. Cervix cancer screening. Lyon: IARC Press; 2005. (IARC Handbooks of Cancer Prevention, 10).

2. Parkin DM, Whelan SL, Feraly J, Teppo L, Thomas DB, editors. Cancer incidence in five continents. v. VIII. Lyon: IARC Press; 2002.

3. Ferenczy A, Franco EL. Persistent human papillomavirus infection and cervical neoplasia. Lancet Oncol 2002; 3:11-6.

4. Schlecht NF, Kulaga S, Robitaille J, Ferreira S, Santos M, Miyamura RA, et al. Persistent human papillomavirus infection as a predictor of cervical intraepithelial neoplasia. JAMA 2001; 286:3106-14.

5. National Institutes of Heath Consensus Development Conference statement on cervical cancer. Gynecol Oncol 1996; 66: 351-61.

6. Winer RL, Lee SK, Hughes JP, Adam DE, Kiviat NB, Koutsky LA. Genital human papillomavirus infection: incidence and risk factors in a cohort of female university students. Am J Epidemiol 2003; 157:218-26

7. Solomon D, Davey D, Kurman R, Moriarty A, O'Connor D, Prey M, et al. The 2001 Bethesda System: terminology for reporting results of cervical cytology. JAMA 2002; 287:2114-9.

\section{Colaboradores}

M. L. Pedrosa participou da realização do trabalho em campo, do processamento e análise dos dados e da redação do artigo. I. E. Mattos e R. J. Koifman colaboraram no delineamento da pesquisa, discussão dos resultados, redação final e revisão crítica do artigo.

\section{Agradecimentos}

Agradecemos à Dra. Lucilia Zardo, diretora do SITEC, pela permissão e apoio para consulta aos bancos de dados que originaram este estudo e a Sheila Prado, funcionária do SITEC que facilitou a busca das informações desejadas.
8. Mount SL, Papillo JL. A study of 10,296 pediatric and adolescent Papanicolaou smear diagnoses in northern New England. Pediatrics 1999; 103: 539-45.

9. Simsir A, Brooks S, Cochran L, Bourquin P, Ioffe OB. Cervicovaginal smear abnormalities in sexually active adolescents: implications for management. Acta Cytol 2002; 46:271-6.

10. Silva CS, Souza MAH, Angelo AG, Pavani RS, Adad J, Murta EFC. Increased frequency of abnormal Papanicolaou smears in adolescents. Arch Gynecol Obstet 2002; 266:154-6.

11. Longatto Filho A, Etlinger D, Gomes N, Cruz SV, Cavalieri MJ. Freqüência de esfregaços cérvicovaginais anormais em adolescentes e adultas: revisão de 308.630 casos. Rev Inst Adolfo Lutz 2003; 62:31-4.

12. Tarkowski TA, Koumans EH, Sawyer M, Pierce A, Black CM, Papp JR, et al. Epidemiology of human papillomavirus infection and abnormal cytologic test results in an urban adolescent population. J Infect Dis 2004; 189:46-50. 
13. Frega A, Stentella P, De Ioris A, Piazze JJ, Fambrini $\mathrm{M}$, Marchionni M, et al. Young women, cervical intraepithelial neoplasia and human papillomavirus: risk factors for persistence and recurrence. Cancer Lett 2003; 196:127-34.

14. Sellors JW, Karwalajtys TL, Kaczorowski J, Mahony JB, Lytwyn A, Chong S, et al. Incidence, clearance and predictors of human papillomavirus infection in women. CMAJ 2003; 168:421-5.

15. Moscicki AB, Schiffman M, Kjaer S, Villa LL. Chapter 5: updating the natural history of HPV and anogenital cancer. Vaccine 2006; 24(3 Suppl):42-51.

16. Winer RL, Kiviat NB, Hughes JP, Adam DE, Lee SK, Kuypers JM, et al. Development and duration of human papillomavirus lesions, after initial infection. J Infect Dis 2005; 191:731-8.

17. Richart RM. A theory of cervical carcinogenesis. Obstet Gynecol Surv 1969; 24(7 Pt 2):874-9.

18. Organização Mundial da Saúde. Problemas de saúde na adolescência. Geneva: Organização Mundial da Saúde; 1965. (Série de Informes Técnicos, 318).

19. Henry MR. The Bethesda System 2001: an update of new terminology for gynecologic cytology. Clin Lab Med 2003; 23:585-603.

20. Edelman M, Fox AS, Alderman EM, Neal W, Shapiro A, Silver EJ, et al. Cervical Papanicolaou smear abnormalities in inner city Bronx adolescents: prevalence, progression, and immune modifiers. Cancer 1999; 87:184-9.

21. Halcon LL, Lifson AR, Shew M, Joseph M, Hannan PJ, Hayman CR. Pap test results among lowincome youth: prevalence of dysplasia and practice implications. J Obstet Gynecol Neonatal Nurs 2002; 31:294-304.

22. Prussia PR, Gay GH, Bruce A. Analysis of cervicovaginal (Papanicolaou) smears, in girls 18 years and under. West Indian Med J 2002; 51:37-9.

23. d'Ottaviano-Morelli MG, Zeferino L, Cecatti JG, Terrabuio DR, Martinez EZ. Prevalence of cervical intraepithelial neoplasia and invasive carcinoma based on cytological screening in the region of Campinas, São Paulo, Brazil. Cad Saúde Pública 2004; 20:153-9.

24. Bleyer A, O'Leary M, Barr M, Ries LAG, editors. Cancer epidemiology in older adolescents and young adults 15 to 29 years of age, including SEER incidence and survival: 1975-2000. Bethesda: National Cancer Institute; 2006.

25. Sadeghi SB, Hsieh EW, Gunn SW. Prevalence of cervical intraepithelial neoplasia in sexually active teenagers and young adults. Am J Obstet Gynecol 1984; 148:726-9.

26. Utagawa ML, Pereira SM, Cavaliere MJ, Maeda MY, Shih LW, Shirata NK. Cervical intraepithelial neoplasia in adolescents: study of cytological findings between 1987 and 1995 in Sao Paulo State-Brazil. Arch Gynecol Obstet 1998; 262:59-64.

27. Ho GYF, Studentsov Y, Hall CB, Bierman R, Beardsley L, Lempa M, et al. Risk factors for subsequent cervicovaginal human papillomavirus infection and the protective role of antibodies to HPV-16 virus-like particles. J Infect Dis 2002; 186:737-42.
28. Chan JK, Monk BJ, Brewer C, Keefe KA, Osann K, McMeekin S, et al. HPV infection and number of lifetime sexual partners are strong predictors for natural regression of CIN 2 and 3. Br J Cancer 2003; 89:1062-6.

29. Leal EAS, Leal Júnior OS, Guimarães MH, Vitoriano MN, Nascimento TL, Costa OLN. Lesões precursoras do câncer de colo em mulheres adolescentes e adultas jovens do Município de Rio Branco - Acre. Rev Bras Ginecol Obstet 2003; 25:81-6.

30. Kahn JA, Rosenthal SL, Succop PA, Ho GYF, Burk $\mathrm{RD}$. The interval between menarche and age of first sexual intercourse as a risk factor for subsequent HPV infection in adolescent and young adult women. J Pediatr 2002; 141:718-23.

31. Moscicki AB, Burt VG, Kanowitz S, Darragh T, Shiboski S. The significance of squamous metaplasia in the development of low grade squamous intraepithelial lesions in young women. Cancer 1999; 85:1139-44.

32. Schiffman M, Kjaer SK. Chapter 2: Natural history of anogenital human papillomavirus infection and neoplasia. J Natl Cancer Inst Monogr 2003; 31: 14-9.

33. Schlecht NF, Platt RW, Duarte-Franco E, Costa MC, Sobrinho JP, Prado JC, et al. Human papillomavirus infection and time to progression and regression of cervical intraepithelial neoplasia. J Natl Cancer Inst 2003; 95:1336-43.

34. Wright JD, Davila RM, Pinto KR, Merritt DF, Gibb RK, Rader JS, et al. Cervical dysplasia in adolescents. Obstet Gynecol 2005; 106:115-20.

35. Monteiro DL, Trajano AJ, Silva KS, Russomano FB. Pre-invasive cervical disease and uterine cervical cancer in Brazilian adolescents: prevalence and related factors. Cad Saúde Pública 2006; 22:2539-48.

36. Moscicki AB, Shiboski S, Hills NK, Powell KJ, Jay N, Hanson EN, et al. Regression of low-grade squamous intra-epithelial lesions in young women. Lancet 2004; 364:1678-83.

37. Kjaer SK, van den Brule AJ, Paull G, Svare EI, Sherman ME, Thomsen BL, et al. Type specific persistence of high risk human papillomavirus (HPV) as indicator of high grade cervical squamous intraepithelial lesions in young women: population based prospective follow up study. BMJ 2002; 325:572.

38. Instituto Nacional de Câncer. Viva mulher. Programa de controle do câncer do colo do útero: manual de implantação do programa. Rio de Janeiro: Instituto Nacional de Câncer; 1997.

39. Saslow D, Runowicz CD, Solomon D, Moscicki AB, Smith RA, Eyre HJ, et al. American Cancer Society guideline for the early detection of cervical neoplasia and cancer. CA Cancer J Clin 2002; 52:342-62.

40. American College of Obstetricians and Gynecologists Committee on Adolescent Health Care. ACOG Committee opinion \#300: cervical cancer screening in adolescents. Obstet Gynecol 2004; 104:885-9.

41. International Agency for Research on Cancer/Alliance for Cervical Cancer Prevention. Planning and implementing cervical cancer prevention and control programs: a manual for managers. Seattle: Alliance for Cervical Cancer Prevention; 2004. 
42. Hildesheim A, Hadjimichael O, Schwartz PE, Wheeler CM, Barnes W, Lowell DM. Risk factors for rapid-onset cervical cancer. Am J Obstet Gynecol 1999; 180 (3 Pt 1):571-7.

43. Karube A, Sasaki M, Tanaka H, Nakagome O, Dahiya R, Fujimoto S, et al. Human papilloma virus type 16 infection and the early onset of cervical cancer. Biochem Biophys Res Commun 2004; 323:621-4.

44. American Academy of Pediatrics. Sexuality education for children and adolescents. Pediatrics 2001; 108:498-502.
45. Agência Nacional de Saúde Suplementar. Caderno de informação da saúde suplementar: beneficiários, operadoras e planos. Rio de Janeiro: Agência Nacional de Saúde Suplementar; 2006.

46. Mirza T, Kovacs GT, McDonald P. The use of reproductive health services by young women in Australia. Aust N Z J Obstet Gynaecol 1998; 38:336-8.

47. Crosby RA, St Lawrence J. Adolescents' use of school-based health clinics for reproductive health services: data from the National Longitudinal Study of Adolescent Health. J Sch Health 2000; 70:22-7.

Recebido em 09/Ago/2007

Versão final reapresentada em 08/Abr/2008

Aprovado em 16/Abr/2008 